\title{
Tight-binding study of bilayer graphene Josephson junctions
}

\author{
W. A. Muñoz, L. Covaci, and F. M. Peeters \\ Departement Fysica, Universiteit Antwerpen, Groenenborgerlaan 171, B-2020 Antwerpen, Belgium \\ (Received 17 July 2012; revised manuscript received 26 September 2012; published 6 November 2012)
}

\begin{abstract}
Using highly efficient simulations of the tight-binding Bogoliubov-de-Gennes model, we solved selfconsistently for the pair correlation and the Josephson current in a superconducting-bilayer graphenesuperconducting Josephson junction. Different doping levels for the non-superconducting link are considered in the short- and long-junction regimes. Self-consistent results for the pair correlation and superconducting current resemble those reported previously for single-layer graphene except at the Dirac point, where remarkable differences in the proximity effect are found, as well as a suppression of the superconducting current in the long-junction regime. Inversion symmetry is broken by considering a potential difference between the layers and we found that the supercurrent can be switched if the junction length is larger than the Fermi length.
\end{abstract}

DOI: 10.1103/PhysRevB.86.184505

PACS number(s): 73.43.-f, 73.23.-b, 73.63.-b

\section{INTRODUCTION}

Ever since graphene, a two-dimensional crystal with honeycomb lattice structure, became recently available, a new variety of hybrid structures has been explored. This led to the development of promising technological devices and the understanding of the nonintuitive physical mechanisms of relativisticlike massless fermions. It is well known that electrons propagating through single-layer graphene (SLG) exhibit many peculiar properties, for example, a gapless linear dispersion around the neutrality (or Dirac) point which resemble massless Dirac fermions, a minimum conductivity at zero carrier concentration, high mobility, and the absence of backscattering. When in contact with superconductors, graphene exhibits exotic superconducting properties. Although graphene was not found to sustain intrinsic superconductivity itself, there is experimental evidence $e^{1-5}$ that when in proximity with a conventional superconductor it becomes superconducting. Because of the conventional superconducting proximity effect, which describes how Cooper pairs diffuse from the superconducting material into metals, superconducting hybrid structures like superconducting-normal-superconducting (SNS) Josephson junctions are interesting systems in which one can study electronic correlation of relativisticlike particles. These experiments have attracted considerable theoretical attention insofar as it was predicted that a finite supercurrent should exist even at zero doping where the density of states is vanishing ${ }^{6}$ or that specular Andreev reflections should happen at the superconductor/graphene interface in SLG. ${ }^{7}$ In the ballistic regime, which is realizable in SNS graphene Josephson junctions according to recent experiments, ${ }^{4,8}$ theoretical studies have shown the existence of a finite bipolar superconducting current through the junction. ${ }^{6,9-12}$

With a gapless parabolic, instead of linear, band structure, bilayer graphene (BLG) appears at this point to be a suitable alternative for investigating electronic correlations in twodimensional systems. Also, as it is widely known theoretically and experimentally, a tunable gap can be induced in BLG by an out-of-plane applied electric field, which is very useful for transistor applications. ${ }^{13,14}$ We expect that the role of the gapless parabolic dispersion may be important since the superconducting correlations depend strongly on the electronic properties of the material. Moreover, the ability to open a gap in the spectrum by an external electric field could be of interest in superconducting devices.

Within a tight-binding Bogoliubov-de-Gennes formalism we calculate self-consistently the pair correlation and the Josephson current through a superconducting-BLGsuperconducting Josephson junction. Our findings resemble previous numerical results shown for SLG-based Josephson junctions except near the Dirac point in case of a long junction, where a suppression of the current is found, mainly due to the vanishing density of states present only in nondimer sites. We also show how the superconducting current can be switched off by applying an out-of-plane electric field.

\section{MODEL}

We consider BLG in the common AB (Bernal) stacking with two inequivalent sublattices $A$ and $B$ in the top layer and the corresponding sublattices $\tilde{B}$ and $\tilde{A}$ in the bottom layer. We model the interlayer interaction with a hopping parameter which couples the nearest neighbors in sublattices $A$ and $\tilde{B}$ from the top and bottom layer. Any additional interlayer coupling terms are ignored in this study. It is well known that in this case the electron dispersion displays two parabolic bands touching at the Dirac point and two additional parabolic bands at higher energies due to interlayer-induced splitting. ${ }^{13,15}$

We study the Josephson effect in a BLG-based junction by considering the hybrid nano-structure illustrated in Fig. 1. Both the top and the bottom layers are in contact with superconducting leads while a junction of size $L$ is suspended. The influence of the superconducting contacts is modeled by assuming an on-site attractive pairing potential, $U_{S}<0$, and heavy doping, $\mu_{S}>0$, in the contact regions, which are labeled by $S$, such that a $s$-wave superconducting state is induced in the outermost regions of both layers. The normal region labeled by $N$, which has a tunable Fermi level $\mu_{N}$ and zero pairing potential, $U_{N}=0$, acts like a non-superconducting channel through which Cooper pairs could tunnel. Similar models for graphene based Josephson junctions were previously considered in the Dirac limit $6,9-11$ as well as in the tight-binding formulation. ${ }^{12,16,17}$ We solve self-consistently for the order parameter along the junction following closely the self-consistent calculation performed in Ref. 12 for a ballistic SLG Josephson junction. This is 


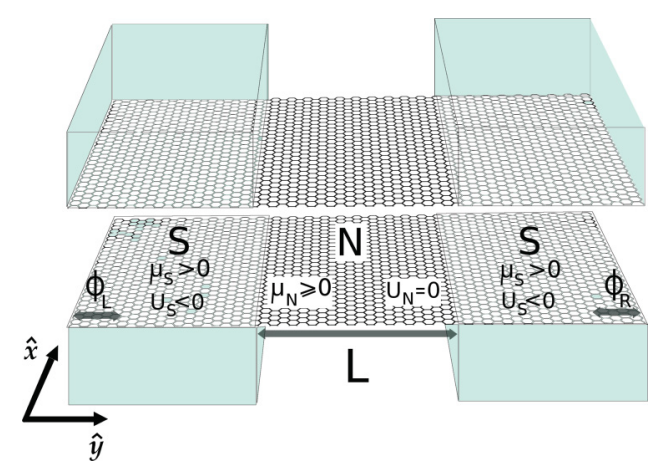

FIG. 1. (Color online) Layout of the SNS-BLG Josephson junction where the superconducting leads are modeled by assuming on-site attractive pairing potential $U_{S}$ and a heavy doping $\mu_{S}$ in the regions under the contacts labeled by $S$. For the non-superconducting link, with length $L$ and labeled by $N$, we took the pairing potential $U_{N}=0$ and a varying chemical potential $\mu_{N}$. Phases $\phi_{L}$ and $\phi_{R}$ are kept fixed during the self-consistent calculation.

necessary in order to consider the possibility of Cooper pairs being depleted close to the interface in the superconducting region due to the existence of the normal region, that is, the inverse proximity effect. As is usually assumed for SLG-based junctions we consider a clean and smooth interface such that all physical quantities are homogeneous along the $\hat{x}$ direction parallel to the interface. In addition, we have considered a wide junction, with width $W \gg L$, as well as periodic boundary conditions imposed along the $\hat{y}$ direction. The latter assumptions make it possible to reduce the self-consistent calculation of the order parameter from a two-dimensional problem to a one-dimensional one since we can restrict the calculation to only a unit cell along the $\hat{y}$ direction perpendicular to the interface. It is worth mentioning that the pairing potential does not distinguish between the sublattices defined on either layer. However, due to the interlayer coupling, the self-consistent calculation should be performed separately for only two inequivalent sites $A$ and $B$ (or $\tilde{A}$ and $\tilde{B}$ ) within the unit cell. The breaking of the inversion symmetry, which is easily achieved by considering a potential difference applied between the layers, makes it necessary to perform the self-consistent calculation for all four sublattice types within the unit cell.

In order to describe the dc Josephson effect we fix the difference in the phases of the order parameter, $\Delta \phi^{S}=\phi_{R}-$ $\phi_{L}$, in the outermost parts of the $S$ regions (see Fig. 1). In a finite region near the interfaces, with size of the order of the coherence length, the order parameter is allowed to relax self-consistently. Therefore, the phase gradient over the nonsuperconducting region $\Delta \phi^{N}$ will be restricted according to $\Delta \phi^{N} \leqslant \Delta \phi^{S} \leqslant \pi$, considering that the maximum value of $\Delta \phi^{S}$ is $\pi$. This constraint for $\Delta \phi^{N}$ was recently pointed out by Black-Shaffer et al. ${ }^{12}$ in a SLG-based ballistic junction.

\section{NUMERICAL METHOD: CHEBYSHEV EXPANSION OF THE GREEN'S FUNCTION}

Superconducting correlations in a BLG-based Josephson junction are described by using the following tight-binding
Hamiltonian with on-site attractive Hubbard interactions:

$$
\begin{aligned}
\mathcal{H}= & -\sum_{\langle i, j\rangle \sigma} t\left(c_{i \sigma}^{\dagger} c_{j \sigma}+\tilde{c}_{i \sigma}^{\dagger} \tilde{c}_{j \sigma}\right)-t_{\perp} c_{i \sigma}^{\dagger} \tilde{c}_{j \sigma} \\
& -\sum_{i \sigma}\left(\mu_{i}+\epsilon_{1}\right) c_{i \sigma}^{\dagger} c_{i \sigma}+\left(\mu_{i}+\epsilon_{2}\right) \tilde{c}_{i \sigma}^{\dagger} \tilde{c}_{i \sigma} \\
& +\sum_{i} U_{i}\left(c_{i \uparrow}^{\dagger} c_{i \uparrow} c_{i \downarrow}^{\dagger} c_{i \downarrow}+\tilde{c}_{i \uparrow}^{\dagger} \tilde{c}_{i \uparrow} \tilde{c}_{i \downarrow}^{\dagger} \tilde{c}_{i \downarrow}\right),
\end{aligned}
$$

where $c_{i \uparrow}^{\dagger}|\mathrm{vac}\rangle$ creates a spin-up electron on the $i$ site in the top layer, whereas $\tilde{c}_{j \downarrow} \mid$ vac $\rangle$ creates a spin-down hole on the $j$ site in the bottom layer. The hopping parameter, $t$, describes the intralayer hopping integral between next-nearest neighbors in the same layers while $t_{\perp}=0.143 t$ corresponds to the interlayer nearest-neighbors hopping which couples the dimer sites $A$ and $\tilde{B}$. Other hopping terms, like the interlayer coupling between the nondimer $B$ and $\tilde{A}$ sites, are not considered in the present work, since they influence only very low-energy excitations. The Fermi level is shifted from the charge neutrality point or Dirac point by the chemical potential $\mu_{i}$ and $U_{i}$ is the on-site attractive pairing potential which is nonvanishing only in the right and left superconducting regions. The on-site energies $\epsilon_{1}$ and $\epsilon_{2}$ for atomic sites on the top and bottom layers, respectively, have been introduced in order to simulate a potential difference or gate voltage $V_{g}=\epsilon_{1}-\epsilon_{2}$ between the layers.

By using the Hartree-Fock decomposition and keeping only terms relevant to the superconducting order, one can transform the many-body Hamiltonian (1) into a mean-field singleparticle Hamiltonian, which within the Nambu formalism can be written as

$$
\mathcal{H}=\sum_{\langle i, j\rangle}\left(c_{i \uparrow}^{\dagger} \tilde{c}_{i \uparrow}^{\dagger} c_{i \downarrow} \tilde{c}_{i \downarrow}\right)\left(\begin{array}{cc}
\hat{\mathcal{H}}_{0} & \hat{\Delta} \\
\hat{\Delta}^{\dagger} & -\hat{\mathcal{H}}_{0}^{\dagger}
\end{array}\right)\left(\begin{array}{c}
c_{i \uparrow} \\
\tilde{c}_{i \uparrow} \\
c_{i \downarrow}^{\dagger} \\
\tilde{c}_{i \downarrow}^{\dagger}
\end{array}\right),
$$

where $\hat{\mathcal{H}}_{0}$ and $\hat{\Delta}$ are the following $2 \times 2$ matrices:

$$
\begin{gathered}
\hat{\mathcal{H}}_{0}=\left(\begin{array}{cc}
\epsilon_{1}+\mu_{i} & 0 \\
0 & \epsilon_{2}+\mu_{i}
\end{array}\right)\left(-\delta_{i j}\right)-\left(\begin{array}{cc}
t & t_{\perp} \\
t_{\perp}^{*} & t^{*}
\end{array}\right)\left(1-\delta_{i j}\right), \\
\hat{\Delta}=\left(\begin{array}{cc}
\Delta_{i} & 0 \\
0 & \tilde{\Delta}_{i}
\end{array}\right) \delta_{i j}
\end{gathered}
$$

where the diagonal elements of the matrix (4) correspond to the on-site mean-field superconducting order parameter $\Delta_{i}=$ $U_{i}\left\langle c_{i \uparrow} c_{i \downarrow}\right\rangle$.

Following Refs. 18 and 19 we have performed the self-consistent mean-field calculation through a numerical approximation of the Gorkov Green's function by using the Chebyshev-Bogoliubov-de-Gennes method. Both the normal and the anomalous Gorkov Green's functions can be approximated by a superposition of a finite number of Chebyshev polynomials as

$$
G_{i j}^{1 \alpha}(\tilde{\omega})=\frac{-2 i}{\sqrt{1-\tilde{\omega}^{2}}}\left[\sum_{n=0}^{N} a_{n}^{1 \alpha}(i, j) e^{-i n \arccos (\tilde{\omega})}\right],
$$


where the expansion coefficients for the diagonal, or normal ( $\alpha=1)$, and the off-diagonal, or anomalous $(\alpha=2)$, components of the $2 \times 2$ Green's function are defined, respectively, as $^{18}$

$$
\begin{aligned}
& a_{i j}^{11}(\omega)=\left\langle c_{i \uparrow}\left|T_{n}(\mathcal{H})\right| c_{j \uparrow}^{\dagger}\right\rangle, \\
& a_{i j}^{12}(\omega)=\left\langle c_{i \downarrow}^{\dagger}\left|T_{n}(\mathcal{H})\right| c_{j \uparrow}^{\dagger}\right\rangle^{*},
\end{aligned}
$$

where $T_{n}(x)=\arccos [n \cos (x)]$ is the Chebyshev polynomial of order $n$, which is defined according to the recurrence relation: $T_{n+1}(x)=2 x T(x)-T_{n-1}(x)$.

In order to perform the sum (5) one needs first to rescale the Hamiltonian (2) such that the eigenvalues lie in the $[-1,1]$ interval. To this end, matrices (2) and (4) as well as the energies have being normalized according to $\mathcal{H} \rightarrow \tilde{\mathcal{H}}=(\mathcal{H}-\mathbb{1} b) / a$ and $\omega \rightarrow \tilde{\omega}=(\omega-b) / a$, where the rescaling factors are $a=$ $\left(E_{\max }-E_{\min }\right) /(2-\eta)$ and $b=\left(E_{\max }+E_{\min }\right) / 2$, with $\eta>0$ being a small number.

Once the Hamiltonian is normalized, the expansion coefficients can be obtained through a recursive procedure. Starting with an initial vector $\left|j_{0}\right\rangle=\left|c_{j \uparrow}^{\dagger}\right\rangle$ and the first term in the iteration $\left|j_{1}\right\rangle=\mathcal{H}\left|j_{0}\right\rangle$ one can obtain the $n$th term by using the Chebyshev recurrence relation: $\left|j_{n}\right\rangle=2 \mathcal{H}\left|j_{n-1}\right\rangle-\left|j_{n-2}\right\rangle$. Chebyshev moments are finally obtained from the scalar product $\left\langle\alpha \mid j_{n}\right\rangle$, where $\langle\alpha|$ are the vectors $\langle 1|=\left\langle c_{i \uparrow}\right|$ and $\langle 2|=\left\langle c_{i \downarrow}^{\dagger}\right|$ for moments expanding the (6) and (7) components of the Green's function, respectively.

Physical quantities, like the local density of states, the pair correlation function, and the Josephson current, can be easily determined once the Green's functions are known:

$$
\begin{gathered}
N_{i}(\omega)=-\frac{2}{\pi} \operatorname{Im} G_{i i}^{11}(\omega), \\
\left\langle c_{i \uparrow} c_{i \downarrow}\right\rangle=\frac{i}{2 \pi} \int_{-E_{c}}^{E_{c}} G_{i i}^{12}(\omega)[1-2 f(\omega)] d \omega,
\end{gathered}
$$

and

$$
J_{i j}=-\frac{1}{\pi} \int\left[i t_{i j} G_{i j}^{11}(\omega)-i t_{i j}^{*} G_{i j}^{11 *}(\omega)\right] f(\omega) d \omega,
$$

respectively. ${ }^{18}$ Since most of the computational effort corresponds to sparse matrix-vector and vector-vector multiplication, high speed-up can be achieved by implementing parallel computation on graphical processing units (GPUs). We are therefore able to solve efficiently systems described by matrices of sizes $N \times N$, where $N$ is between $88 \times 10^{3}$ and $320 \times 10^{3}$, according to the junction size considered in this study. Note that for system size of this order the direct diagonalization of the Hamiltonian matrix is not possible. Additional parallel computations can be implement by considering that all physical quantities calculated here, such as density of states, pair correlation, and the Josephson current, can be obtained from the Green's function for each lattice point separately.

\section{RESULTS}

It is well known that the energy dispersion in SLG and BLG differ around the neutrality point. Therefore, qualitative differences are expected in the proximity effect as well as in the Josephson current in the two systems. For a quantitative comparison between SLG and BLG Josephson junction we have set up the following values for the physical input parameters: $U_{S}=-1.36 t=-3.4 \mathrm{eV}$ and $\mu_{S}=0.6 t=$ $1.5 \mathrm{eV}$, similar to the values used in a previous self-consistent study for ballistic SLG Josephson junctions. ${ }^{12}$ These parameters lead to a finite $s$-wave bulk superconducting order parameter, $\Delta_{0}=0.041 t$, which corresponds to a superconducting coherence length $\xi=\hbar v_{F} / \Delta_{0} \approx 33 a$, where $a$ is the $\mathrm{C}-\mathrm{C}$ interatomic distance, similar to the one considered in the SLG junction case. Due to the difference in the local density of states between dimer and nondimer locations, the order parameter is slightly different for these two types of atoms. Both junction-length regimes are solved with the proposed self-consistent numerical method, for example, short junction for $L<\xi$ and long junction for $L>\xi$. For both cases the width of the samples has been chosen as the minimal value of the ratio $W / L$ for which no important variations of the pair correlation are observed when increasing the width of the junction. For short and long junctions it was found that $W / L=0.05,0.1$, respectively, were necessary in order to avoid a relevant width dependence of the results.

Previous analytical descriptions of BLG Josephson junctions based on the Dirac equation ${ }^{10,20}$ require smooth interfaces and a low-energy regime, for which $\Delta_{0} \ll t_{\perp} \ll \mu_{S}$. Here these restrictions are lifted, but in order to compare with relevant experimental scenarios we performed calculations only for situations corresponding to $\Delta_{0}<t_{\perp}<\mu_{S}$.

\section{A. Proximity effect}

We show in Fig. 2 the self-consistently calculated pair correlation $\left\langle c_{i \uparrow} c_{i \downarrow}\right\rangle$ for both inequivalent sites, $A$ (dimer) and $B$ (nondimer), in a unit cell defined along the $\hat{y}$ direction perpendicular to the interface. As was previously mentioned, both long and short junctions are considered here and plotted in Figs. 2(a) and 2(b), respectively. We present the pair correlation function for several doping levels in the non-superconducting region, showing that the proximity effect is strongly dependent on the relative Fermi level mismatch (FLM) between the $S$ and $N$ regions. A significant difference between BLG and SLG can be seen for the undoped case $\left(\mu_{N}=0\right)$ while for other dopings the pair correlation exhibits similar behavior in BLG and SLG. In particular, we can see that in the undoped case dimer sites in BLG show a suppression of the pair correlation over the $N$ region compared to the SLG case. Opposite behavior is seen for nondimer sites where a larger pair leakage into $N$ is found. This behavior is similar to the proximity effect in strained graphene where a sublattice polarization of the local density of states in the zeroth pseudo-Landau level induces sublattice-dependent leaking distances. ${ }^{17}$ No relevant differences in the pair correlation profile are found between dimer and nondimer sites in BLG for the higher doping levels considered here: moderately doped $\mu_{N}=0.3 t$ and highly doped $\mu_{N}=0.6 t$. Note also in Fig. 2(b) that the reported interlayer asymmetry in the pair correlation in BLG is found to be not important for the short-junction regime. In fact, we can clearly see in Fig. 2(c) that the difference between the pair correlation at sites $A$ and $B$ becomes larger as the junction length is increased, as large as a few orders of magnitude. 

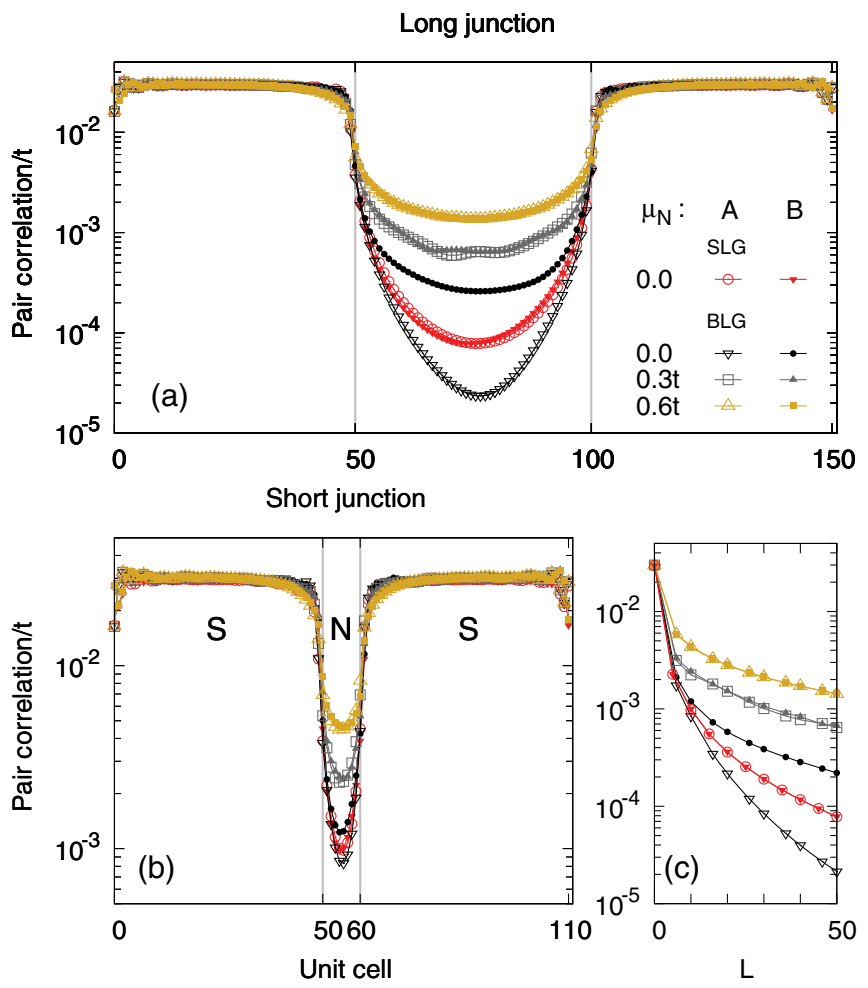

FIG. 2. (Color online) Absolute value of the pair correlation in a long (a) and short (b) BLG Josephson junction as a function of the position along the $\hat{y}$ direction perpendicular to the $S N$ interface. Both inequivalent sites, dimer at $A$ and nondimer at $B$, are plotted separately for different doping levels considered for the non-superconducting region: $\mu_{N}=0,0.3 t$, and $0.6 t$. The first one corresponds to the case when the Fermi level is pinned at the Dirac point while the last one corresponds to no FLM at the interface. (c) Pair correlation at $L / 2$ in the $N$ region as a function of the junction length $L$ for different values of $\mu_{N}$. SLG self-consistent results are shown for comparison with the BLG undoped case.

\section{B. LDOS}

To further understand this peculiar behavior observed only in the undoped case we plot the local density of states (LDOS) in the superconducting and non-superconducting regions. The LDOS is plotted for dimer $(A)$ and nondimer $(B)$ lattice sites across the junction in the long-junction limit where we have previously found differences in the proximity effect between SLG and BLG Josephson junctions. Three particular cases have been chosen and shown in the top panels of Fig. 3 . Panel (a) shows the LDOS for two sites in the middle of the $N$ region for the undoped case, whereas, in panel Fig. 3(b) we have depicted the LDOS for the same locations but when there is no FLM; that is, the doping is the same throughout the graphene layer. Results shown in Figs. 3(a) and 3(b) are consistent with the fact that the density of states at dimer sites vanishes linearly around the Dirac point, while being finite at the nondimer sites, ${ }^{13,15}$ as this can be clearly seen in the inset of Fig. 3(b). Due to the differences in the LDOS for inequivalent sites in BLG, the proximity effect will give different leaking distances in different sublattices, as we show in Fig. 2(c) for the undoped case. In fact, the inset of Fig. 3(b)
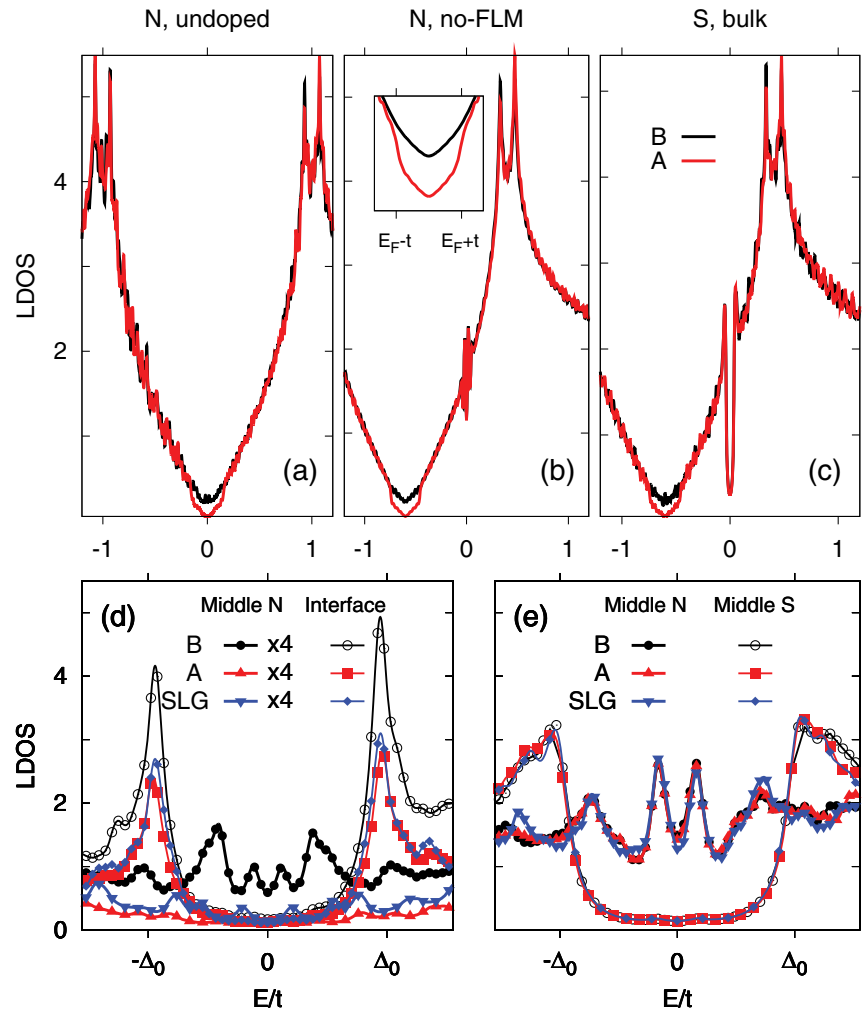

FIG. 3. (Color online) LDOS for A (red) and B (black) sublattice sites in the $N$ region with (a) zero doping (at the Dirac point) and (b) heavy doping (or with no-FLM between the superconducting and normal regions). (c) LDOS in the highly doped $S$ region showing the superconducting gap. The inset in panel (b) emphasizes the difference in the LDOS between A and B sublattice: a vanishing and finite density of states around the Dirac point. A magnification of (a) around the Dirac point is shown in panel (d), as well the LDOS of atomic sites at the interface for both sublattices. A close up view of the panels (b) and (c) around the zero energy is shown in (e) where the superconducting gap in the $S$ region and the Andreev states in the highly doped $N$ are discernible. SLG analogous cases (blue) have been included for comparison purposes. Note that the results presented here correspond to the long-junction limit.

shows a better appreciation of the LDOS of Fig. 3(b) around the Dirac point where we notice the formation of Andreev states for a nondimer site in the middle of the undoped $N$ region. For the cases of dimer sites and for SLG, where we have a vanishing density of states at the Dirac point, we find that the Andreev peaks are strongly suppressed. In addition, we show in Fig. 3(d) the energy gap at the interface where the coherence peaks are more pronounced at the nondimer sites in comparison to the dimer sites and the SLG junction.

Increasing the doping level in the normal region makes the LDOS more homogeneous. This result is consistent with the fact that no difference between both kind of sites is observed for the other doped cases in the pair correlation function shown in the previous section. Finally, Fig. 3(c) shows the LDOS deep inside the superconducting region where we can clearly see the coherence peaks on each side of the superconducting gap. Note that the LDOS becomes homogeneous in both sublattices and resembles the one of SLG one for the highly doped case, and 

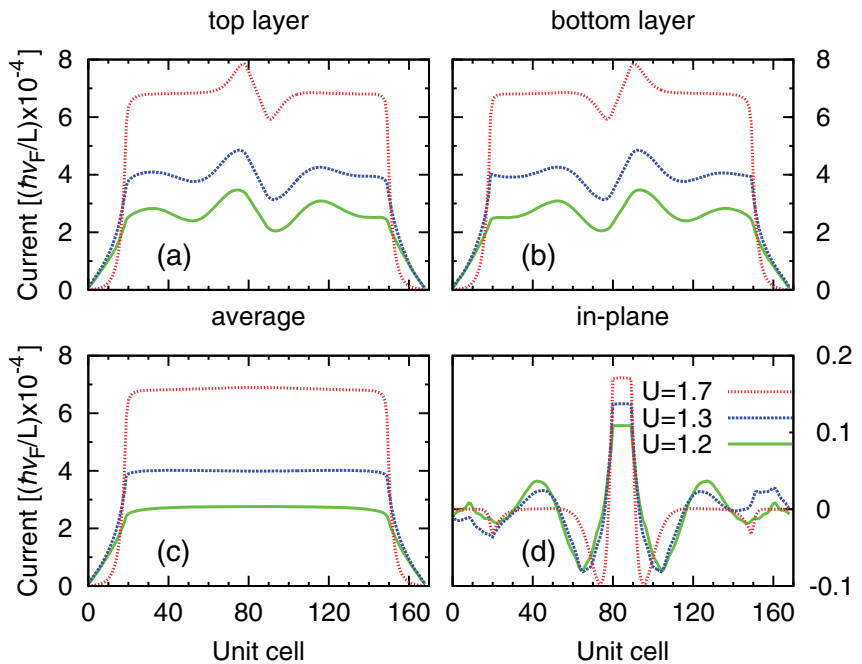

FIG. 4. (Color online) Self-consistent Josephson current as a function of position along the $\hat{y}$ direction for top (a) and bottom (b) layer as well as the average current (c) in the system for different values of the pairing potential, that is, different coherence lengths. The interlayer current between the $A-\tilde{B}$ dimer sites is plotted in (d).

no difference is observed in the superconducting gap even in the zoomed-in view shown in Fig. 3(e) for the $N$ and $S$ region.

\section{Josephson current}

\section{Unbiased case}

In the absence of applied bias voltage but in the presence of a finite phase difference between the two superconducting sides, a dc supercurrent will flow across the junction. ${ }^{12,21}$ This is the usual dc Josephson effect. For this purpose a phase bias is achieved by fixing a desired phase difference between the outermost parts of the superconducting regions, $\phi_{L}$ and $\phi_{R}$ for the left and right sides of the junction, respectively (see Fig. 1). In order to numerically calculate the Josephson current we solve self-consistently for both phase and amplitude of the order parameter along the junction except in the extreme regions where we keep the phases fixed. The current profile along the junctions in both layers as well as the interlayer current are shown in Figs. 4(a)-4(d) for an undoped non-superconducting link for different values of the pairing potential. As we can see, the supercurrent is found not to be constant within each layer separately, contrary to what is usually expected to happen for self-consistent current calculation in two-dimensional systems. ${ }^{21}$ Instead, one interesting feature in BLG is the appearance of a weak interlayer current between $A-\tilde{B}$ dimer sites as a consequence of the current conservation law. We observe that the LDOS at the left interface is asymmetric in top-bottom layers while for the right interface the asymmetry is reversed. Because of this, the current is enhanced at the left interface in the top layer while being suppressed in the bottom layer; therefore, a weak interlayer current appears. The reverse happens at the right interface, but the average current remains flat across the whole junction, as expected. Next we construct the current-phase relation (CPR) by performing self-consistent current calculations for different
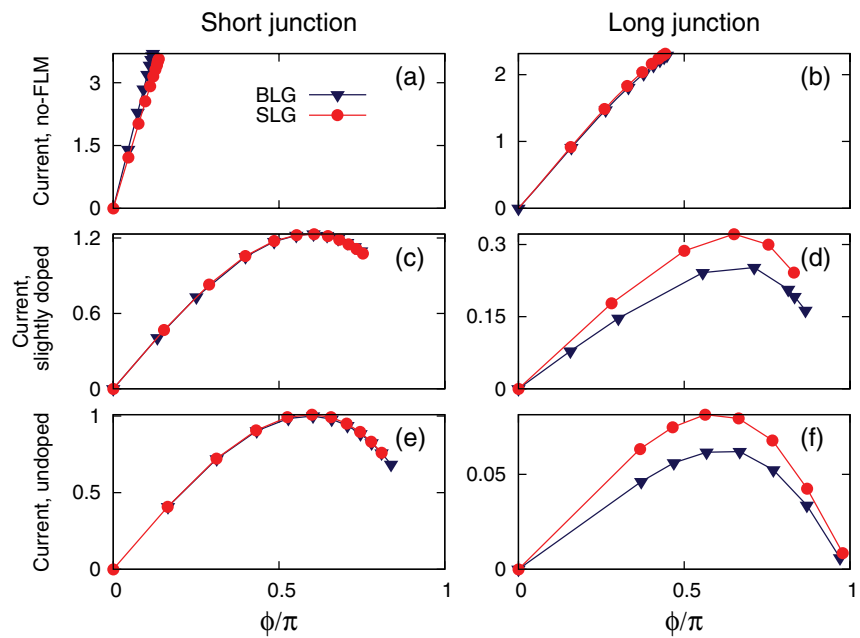

FIG. 5. (Color online) Current-phase relation normalized to the critical current found in (e) for SLG- and BLG-based junctions considering different doping levels in the $N$ region: (e), (f) undoped $\mu_{N}=0$; (c), (d) slightly doped $\mu_{N}=0.1 t$; and (a), (b) no-FLM $\mu_{N}=\mu_{S}=0.6 t$ for short (a), (c), (e) and long (b), (d), (f) junctions. The short- and long-junction lengths are $L=10$ and $L=50$, respectively.

phase differences between the superconducting contacts and doping levels in the non-superconducting region. We show in Fig. 5 the phase dependence of the current for both SLG and BLG for no-FLM cases with $\mu_{N}=0.6 t$ [panels (a) and (b)], slightly doped with $\mu_{N}=0.1 t<t_{\perp}$ [panels (b) and (c)], and undoped [panels (e) and (f)]. We consider both regimes, short junctions [panels (a), (c), and (e)] and long junctions [panels (b), (d), and (f)]. Note that, in most of the cases shown in Fig. 5(a) complete description of the current over the full $[0, \pi]$ phase range is not possible. This restriction appears as a consequence of the relaxation of the phase over the $S$ region which becomes significant as the FLM is reduced in the junction until $S$ and $N$ regions are equally doped and the phase drop goes linearly through the self-consistent region. Therefore, the phase difference over the normal region will always be smaller than the applied phase difference in the superconducting regions. A similar constraint is found for SLG-based junctions, as is shown in Fig. 5 and which was previously pointed out by Black-Schaffer et al. ${ }^{12}$ We find that for no-FLM situations the Josephson current density and the CPR of the BLG is almost identical to the one in SLG for both short and long junctions. In contrast, for the undoped and slightly doped cases the short and long junctions have different behavior. Short BLG junctions are similar to short SLG ones, while for long BLG junctions the Josephson current is suppressed. The origin of this suppression could be traced back to the sublattice polarization of the leaking distance which remains even for doping levels lower than the interlayer hopping energy, $t_{\perp}$. While one sublattice (nondimer sites) has an enhanced leaking distance, the other sublattice (dimer sites) behaves like an insulator with a short leaking distance. The resulting combination corresponds to a slightly suppressed Josephson current when compared to SLG. 

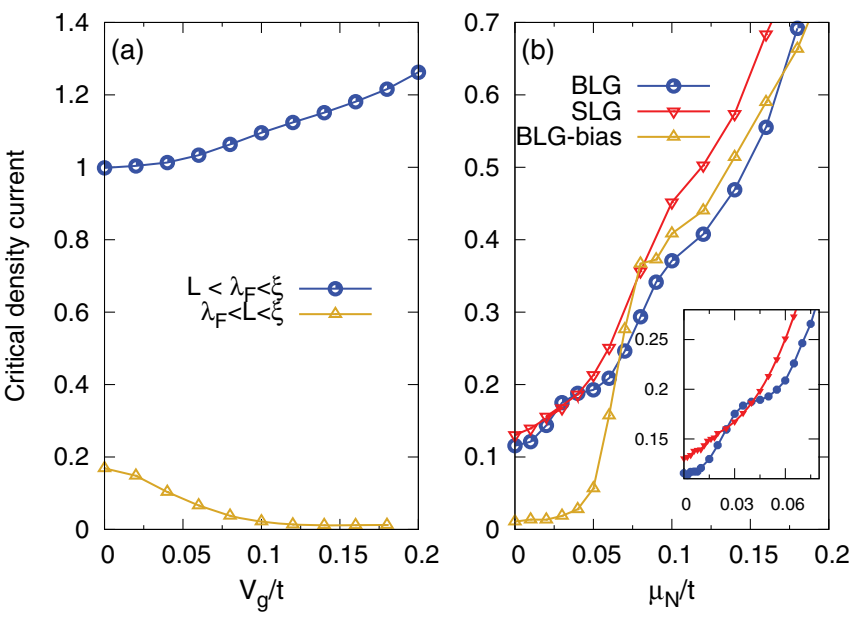

FIG. 6. (Color online) Critical density current in a short BLG junction, with $L=40 a$ and $U_{S}=-1.2 t$, as a function of the gate voltage $V_{g}$ at the Dirac point (a) and the chemical potential $\mu_{N}$ (b). In the left panel two different regimes are considered, $L<\lambda_{F}$ and $L>$ $\lambda_{F}$, showing an enhancement and suppression of the superconducting current, respectively. In the right panel $\mu$ dependence is plotted for bias and unbias cases. SLG is included for comparison.

\section{Biased case}

Inversion symmetry can be broken in the BLG nanostructure by considering a potential difference, $V_{g}=\epsilon_{1}-\epsilon_{2}$ with $\epsilon_{1}=V_{g} / 2$ and $\epsilon_{2}=-V_{g} / 2$, applied between the layers. As a consequence, a tunable gap $\Delta_{0}$ is induced at the Dirac point for the undoped case and therefore inversion symmetry breaking appears as a good possibility to switch off the superconducting current when the voltage-induced gap overtakes the superconducting gap, $\Delta_{g}>\Delta_{0}$. At this point, we consider only short junctions where such proposal might have potential technological applications. In Fig. 6(a) we show the dependence of the supercurrent on the applied bias voltage, $V_{g}$, perpendicular to the BLG layer. We observe two distinct regimes, according to the relation between the Fermi wavelength and the junction length. In the case when $\xi>\lambda_{F}=\hbar v_{F} /\left(V_{g} / 2\right)>L$, we observe an enhancement of the Josephson current. This is because the charge density in the junction does not recover its bulk expected value when the BLG is under bias ( $+n$ for one layer and $-n$ in the other layer) but has a finite positive value. In this regime the junction is effectively doped, thus showing an enhancement of the current. A different dependence of the current is achieved when $\xi>L>\lambda_{F}$. In this case for the center of the junction the charge density has the opposite polarity, and the expected suppression of the current with bias voltage is obtained.

In Fig. 6(b) we show the $\mu$ dependence of the current for the biased and unbiased cases. A doping activation is found in the bias case for an energy value around $\Delta_{g}$; in addition, a slight increase in the current is observed at $\mu_{N} \approx \Delta_{g}$ due to the enhancement of the LDOS above the gap edge. SLG results are included in order to compare directly with BLG and show that for the chosen length the current density is slightly larger in SLG. In the inset of Fig. 6(b) we focus on the low doping regime and observe that in BLG additional oscillations appear, reminiscent of what was previously found analytically in the Dirac approximation. ${ }^{10}$

\section{CONCLUSIONS}

In conclusion, by using an efficient numerical procedure we have solved self-consistently the Bogoliubov-de-Gennes equations for a tight-binding model of the AB-stacked BLG Josephson junction. When compared to SLG Josepshon junctions we uncover several regimes. First, in the short-junction regime, the current density is similar for SLG and BLG for any doping of the normal junction region. Second, in the long-junction regime, for undoped junctions, the BLG current density is suppressed while for doped junctions (with doping larger than $t_{\perp}$ ) the BLG and SLG junctions behave in a similar way. We attribute the peculiar behavior of the undoped BLG junctions to the difference of the LDOS between the dimer and nondimer sites, which give suppressed or enhanced Cooper pair leaking distances depending on the sublattice. We have calculated the CPR and showed that, similar to SLG, even for short junctions there is a departure from conventional symmetric CPR. Finally, we have shown that by applying a gate voltage perpendicular to the BLG a gap in the spectrum can be induced and a supercurrent switch can be achieved given that the junction length is larger than the Fermi wavelength.

\section{ACKNOWLEDGMENT}

This work was supported by the Flemish Science Foundation (FWO-Vl).
${ }^{1}$ A. Kanda, T. Sato, H. Goto, H. Tomori, S. Takana, Y. Ootuka, and K. Tsukagoshi, Physica C 470, 1477 (2010).

${ }^{2}$ H. Tomori, A. Kanda, H. Goto, S. Takana, Y. Ootuka, and K. Tsukagoshi, Physica C 470, 1492 (2010).

${ }^{3}$ H. B. Heersche, P. Jarillo-Herrero, J. B. Oostinga, L. M. K. Vandersypen, and A. F. Morpurgo, Nature (London) 446, 56 (2007).

${ }^{4}$ X. Du, I. Skachko, and E. Y. Andrei, Phys. Rev. B 77, 184507 (2008).

${ }^{5}$ C. Ojeda-Aristizabal, M. Ferrier, S. Guéron, and H. Bouchiat, Phys. Rev. B 79, 165436 (2009).
${ }^{6}$ M. Titov and C. W. J. Beenakker, Phys. Rev. B 74, 041401 (2006).

${ }^{7}$ C. W. J. Beenakker, Phys. Rev. Lett. 97, 067007 (2006).

${ }^{8}$ F. Miao, S. Wijeratne, Y. Zhang, U. C. Coskun, W. Bao, and C. N. Lau, Science 317, 1530 (2007).

${ }^{9}$ I. Hagymási, A. Kormányos, and J. Cserti, Phys. Rev. B 82, 134516 (2010).

${ }^{10}$ M. Alidoust and J. Linder, Phys. Rev. B 84, 035407 (2011).

${ }^{11}$ K. Halterman, O. T. Valls, and M. Alidoust, Phys. Rev. B 84, 064509 (2011). 
${ }^{12}$ A. M. Black-Schaffer and S. Doniach, Phys. Rev. B 78, 024504 (2008).

${ }^{13}$ A. H. Castro Neto, F. Guinea, N. M. R. Peres, K. S. Novoselov, and A. K. Geim, Rev. Mod. Phys. 81, 109 (2009).

${ }^{14}$ E. V. Castro, K. S. Novoselov, S. V. Morozov, N. M. R. Peres, J. M. B. L. dos Santos, J. Nilsson, F. Guinea, A. K. Geim, and A. H. Castro Neto, Phys. Rev. Lett. 99, 216802 (2007).

${ }^{15}$ F. Guinea, A. H. Castro Neto, and N. M. R. Peres, Phys. Rev. B 73, 245426 (2006).
${ }^{16}$ A. M. Black-Schaffer and J. Linder, Phys. Rev. B 82, 184522 (2010).

${ }^{17}$ L. Covaci and F. M. Peeters, Phys. Rev. B 84, 241401 (2011).

${ }^{18}$ L. Covaci, F. M. Peeters, and M. Berciu, Phys. Rev. Lett. 105, 167006 (2010).

${ }^{19}$ A. Weiße, G. Wellein, A. Alvermann, and H. Fehske, Rev. Mod. Phys. 78, 275 (2006).

${ }^{20}$ I. Snyman and C. W. J. Beenakker, Phys. Rev. B 75, 045322 (2007).

${ }^{21}$ L. Covaci and F. Marsiglio, Phys. Rev. B 73, 014503 (2006). 